\title{
Real-time protection of in-vessel components in ASDEX Upgrade
}

\author{
A. Herrmann, R. Drube, T. Lunt, P. de Marné and ASDEX Upgrade team \\ Max-Planck-Institut für Plasmaphysik, EURATOM Association, D-85748 Garching, Germany
}

\begin{abstract}
A video real time safety system (VRT) for protection of in-vessel components was fully implemented in the machine control system (CODAC) from the 2007 experimental campaign on. The VRT is based on video cameras in contrast to infrared systems. The visible wavelength range has a smaller measurement range but is a factor 5-10 less sensitive against changes of the transmission of the optical system and the target emissivity compared to infrared systems. Up to 12 analog video channels with multiple regions of interest (ROI) are processed and monitored on each video stream. At present two safety algorithms, to detect the fraction of overheating in a ROI and hot spot detection, respectively, are implemented. The integral algorithm is preferentially used for probe or limiter protection, the hot spot algorithm for divertor protection. The VRT system is realized with ReadHawk real time operating system on a multi core Linux computer.
\end{abstract}

Keywords: ASDEX Upgrade, Image processing, Real-time, safety system, wavelength selection

\section{Introduction}

The fusion experiment ASDEX Upgrade (AUG) is a mid-size tokamak well suited for investigation of plasma wall interaction and heat exhaust for future reactor size experiments [1]. Safe operation of AUG needs a protection of in-vessel components against overheating that can occur mainly due to the following reasons. (i) The available heating power of up to $20 \mathrm{MW}$ neutral beam heating and $8 \mathrm{MW}$ of wave heating allows experiments with an ITER relevant $\mathrm{P}_{\text {heat }} / \mathrm{R}_{\text {maj }}$ of up to 17 $\mathrm{MW} / \mathrm{m}$. The resulting divertor power load in combination with pulse lengths of 5-10 s exceeds the divertor energy limit of about $20 \mathrm{MJ}$. The corresponding ELM averaged peak heat load can be well above 10 $\mathrm{MW} / \mathrm{m}^{2}$ resulting in target temperatures above $2000^{\circ} \mathrm{C}$ for long pulses. (ii) The evolution of AUG from a carbon to a full tungsten experiment was finished in 2007 with the installation of graphite divertor plates coated with $200 \mu \mathrm{m}$ tungsten. These coated graphite tiles were qualified for an ELM averaged cyclic load of $10 \mathrm{MW} / \mathrm{m}^{2}$ and single events up to $23 \mathrm{MW} / \mathrm{m}^{2}$. Exceeding these limits resulted in a delamination of the tungsten coating and eventually a disruption of the discharge. (iii) Reciprocating probes are used to measure plasma parameters in the scrape-off layer (SOL). Here the heat flux can reach values of a few $100 \mathrm{MW} / \mathrm{m}^{2}$ resulting in a significant overheating of exposed probes if their exposition time is too long for the preprogrammed separatrix-probe distance. As a result the probe heads can be destroyed and broken parts can fall down into the divertor region [2].

The failures caused by overheating of in-vessel components have not the character of a high level incident, as it would be e.g. the loss of a toroidal field coil or the vessel support, but forces a stop of experimental operation and an unscheduled vessel venting for repair, followed by baking and machine conditioning to come back to normal plasma operation. Usually, this procedure needs a few weeks that are lost for the physics program. This was the motivation to install a first real time video safety system at AUG in 2002. This system was redesigned and upgraded in 2007 to meet the needs of a full tungsten first wall.

This paper will discuss the advantages and disadvantages of a machine protection system based on video cameras compared to infrared cameras as they are used in Tore Supra [3] and projected for ITER [4]. In addition, the hard and software and its interaction with the CODAC system [5] will be described in the following section. In section 3 examples for the action of the VRT will be presented and its reliability will be discussed. Finally, a summary is given in section 4

\section{The ASDEX Upgrade Video real-time safety system}

The video real-time system (VRT) for machine protection is based on CCD/CMOS cameras installed in different ports of AUG covering completely the lower divertor (see Fig. 1) and the outboard protection limiters and nearly the whole of the remaining in-vessel surface.

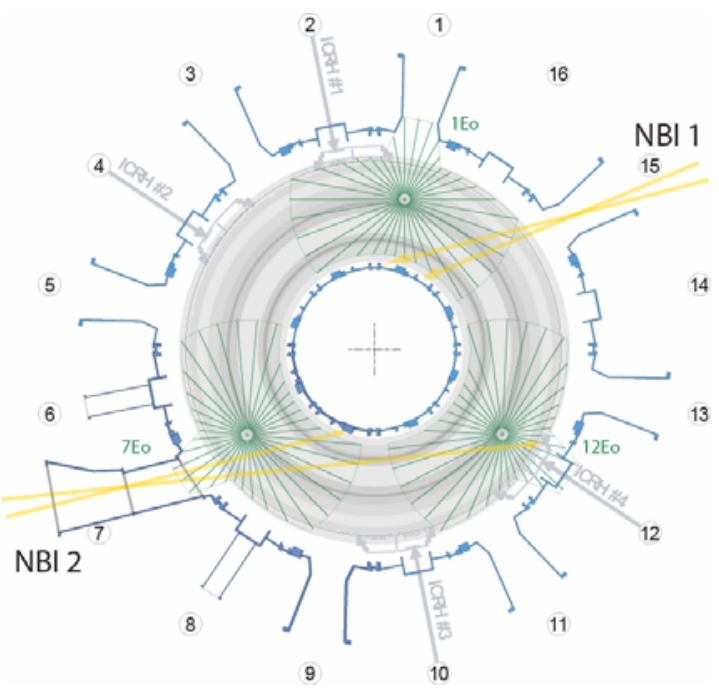

Fig. 1 Arrangement of top-down looking video diagnostics at AUG. The cameras 1Eo, 7Eo and 12 Eo ensure the view to the whole lower divertor. 
The use of the visible wavelength range instead of the infrared region has a few cons and pros that are discussed in the following subsection before the VRT system is presented in detail and the implemented safety algorithms are discussed.

\subsection{Video vs. infrared cameras}

Optical surface temperature measurement is based on Planck's law that gives the relation between the power/photons emitted from a surface and its temperature [6]. The detector signal, $\mathrm{S}_{\mathrm{det}}$, is given by the signal due to the thermal emission of the target, $S_{\text {target, }}$ and a background signal due to the optical transmission of the system, $S_{\mathrm{bck}}$, that is assumed to be constant during the measuring period, $S_{\mathrm{det}}=S_{t \text { arg } e t}+S_{b c k}$

with $S_{\text {target }}=K \varepsilon \tau /\left(\exp \left(\frac{c_{2}}{\lambda T}\right)-1\right)$. Here $\mathrm{S}_{\text {target }}$ represents the temperature dependent part of Planck's law. The wavelength dependent part of Planck's law that is constant for a given detector system is included in the calibration factor K. $\tau$, is the transmission of the system and, $\varepsilon$, the emissivity of the target. $\mathrm{c}_{2}$ is related to elementary constants, $c_{2}=c h / k=14380 \mu \mathrm{m} \mathrm{K}$.

Fig. 2 shows exemplarily the signals for the main wavelength regions used for optical temperature measurements, visible/near infrared (vis/NIR, $\lambda=0.9-$ $1.1 \mu \mathrm{m}$ ) mid wave infrared (MWIR, $\lambda=4-5 \mu \mathrm{m}$ ), and long wave infrared (LWIR, $\lambda=9-11 \mu \mathrm{m}$ ) together with the noise level and a typical saturation limit for detectors with about 3-4 orders of magnitude of dynamic range. It is obvious that MWIR and LWIR can cover the complete temperature range from 500 to $3500 \mathrm{~K}$ without adjustment of the measuring parameters. The signal of LWIR systems is significantly higher in the low temperature range, in particular at room temperature, compared to MWIR and vis/NIR systems. But LWIR systems have no real advantage for temperature measurements starting at moderate temperatures of about $500 \mathrm{~K}$ as required by ITER because the dynamic range of the detector is not exploited. Here MWIR systems have the advantage of a signal above the noise level in combination with the exploitation of the dynamic range.

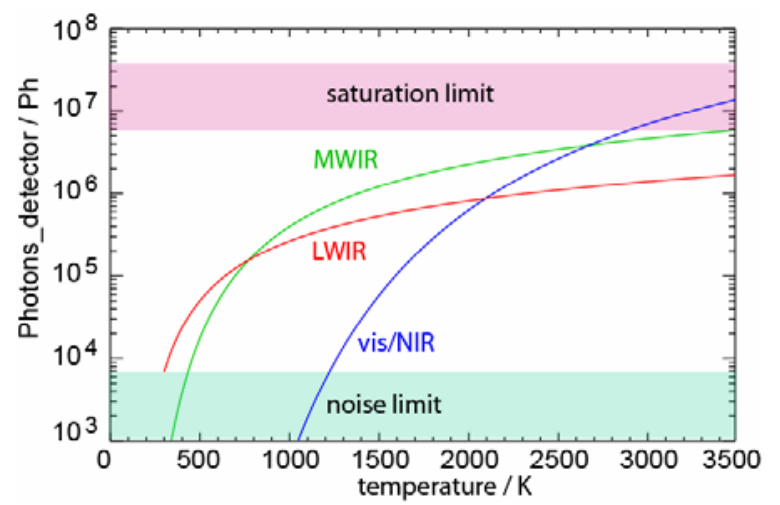

Fig. 2 Detector signal for vis/NIR, MWIR, LWIR wavelengths regions (f-number: $F / \#=8$, pixel size $30 \mu \mathrm{m}^{2}, \tau=\varepsilon=1,1 \mu \mathrm{s}$ integration time, bandwidth $\Delta \lambda= \pm 0.1 \lambda$.
The lower limit for temperature measurement with Vis/NIR systems is at about $1200 \mathrm{~K}$. The change of the signal with increasing temperature (sensitivity) is significantly higher compared to MWIR/LWIR systems resulting in a saturation of the system at about $2800 \mathrm{~K}$, This measurable temperature interval of $\Delta \mathrm{T}=1600 \mathrm{~K}$ is rather narrow compared to that of MWIR and LWIR systems. The relative error of the temperature measurement can be calculated from Equ 1:

$$
\frac{\Delta T}{T}=\frac{\lambda T}{c_{2}}\left(\frac{\Delta K}{K}+\frac{\Delta \varepsilon}{\varepsilon}+\frac{\Delta \tau}{\tau}+\frac{S_{b c k}}{S_{\text {target }}} \frac{\Delta S_{b c k}}{S_{b c k}}\right)
$$

Fig. 3 shows the relative error in dependence on the target temperature. The factor in front of the sum in Equ (2) mitigates the relative error of calibration, optical transmission and emissivity of the target in the vis/NIR wavelength region by a factor of 5-10 compared to the MWIR and LWIR region. It is about $0.1 @ 1500 \mathrm{~K}$ and $0.2 @ 3000 \mathrm{~K}$, i.e. well below unity also at the upper end of the temperature measurement region. The disadvantage of the vis/NIR region with respect to the measurement range as shown in Fig. 2 is an advantage for the measurement error (Fig. 3). This comparator like behavior of a vis/NIR temperature measurement in combination with robustness against change of system parameters like emissivity and transmission, in particular for a tungsten surface is exploited for the machine protection system in AUG.

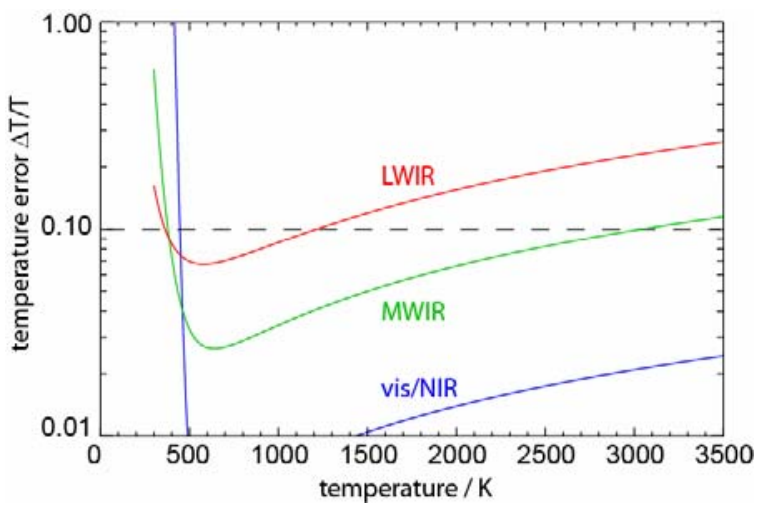

Fig. 3 Relative temperature error for a system with a signal to noise ratio of 0.1 at the background temperature and $10 \%$ error for the sum of emissivity, transmission and calibration (dashed line).

\subsection{The VRT system}

A first real time safety system with two channels was implemented in 2002 mainly for protection of limiters and probes. A new significantly improved system came into operation in 2007 together with the start of the full tungsten experiments. It aims on the following topics [7]:

- Replacing of the old video recorder based monitoring system. Digitizing of 12 video channels with 8 Bit ADCs and storing into MPEG2 data format.

- Synchronizing with the discharge time by adding a time stamp (time of arrival/grabbing) for each full frame.

- Direct integration of the VRT system as a safety system into the new AUG fast control system [5] by implementing a real-time signal exchange protocol and synchronizing the activities between the two systems. 
- Selection of ROIs with arbitrary shape for up to 32 ROIs in total for all channels.

- Keeping the response time in the order of a full frame time, i.e. about $40 \mathrm{~ms}$.

- Realising of four stacked alarm limits that would allow an event dependent reaction of the CODAC system, e.g. change the plasma position, reduce the heating power, and increase the density or shutdown of the discharge.

A principal scheme of the realization and implementation of the system is illustrated in Fig. 4. The VRT hardware is realized with Matrox Morphis frame grabber cards to digitize the 12 analog video signals. Four Xeon Paxville 7030 MP microprocessors running at $2.8 \mathrm{GHz}$ with dual core and hyper-threading technology offer 16 virtual CPUs. Each of the three Morphis frame grabber cards is assigned to one CPU for interrupt handling. The VRT operating system is RedHawk [8]. It guarantees that a user-level application can respond to an external event in less than 15 microseconds on most hardware platforms.

The connection to the discharge control system is via a status signal, reporting periodically $(40 \mathrm{~ms})$ the status of the processing pipelines (watchdog monitoring of each video channel) and the ROI-monitor status.

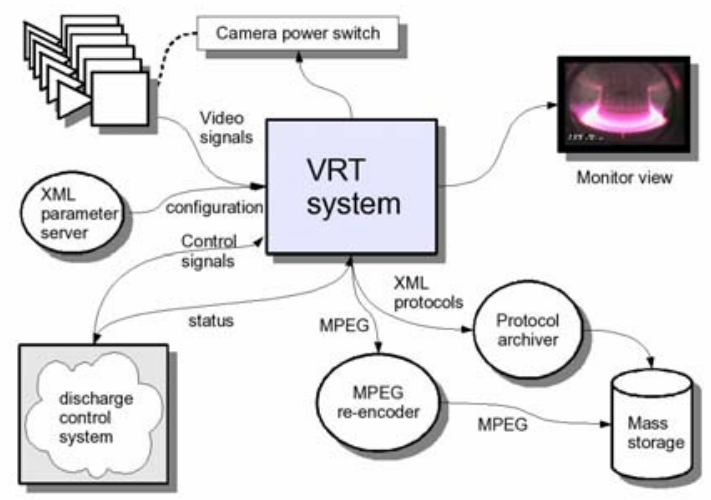

Fig. 4 Data flow diagram of the real time video system.

The configuration for every discharge is done by an XML file. ROIs are also defined as separate XML descriptions. Different ROIs may overlap. Also, any ROI can be composed of possibly disjoint parts of the whole picture. For each ROI one or more monitors can be defined in the XML configuration file, each characterized by an optional filter, a detection algorithm, an alarm threshold, and an activation switch for local deactivation.

Additionally one video channel can be selected to be displayed on a large plasma monitor in the control room.

The MPEG 2 files and the XML protocol files produced by the VRT system during the discharge are stored in an archive and are accessible for off-line discharge inspection.

\subsection{Video processing and real-time algorithms}

As mentioned in the previous subsection, monitors can be defined for each ROI.
Incoming grabbed video data are treated first by a pre-processing routine. First, a background correction is performed with frames stored before the plasma discharge and the dynamic range of the detector is normalized from zero (black) to one (white). Optional, the effect of neutrons on the detector can be eliminated by a median filter with the width 3 . This removes single pixels with a strong brightness.

These pre-processed data are then used by the detection algorithms. The first algorithm measures the integrated intensity, i.e. the fraction of pixels in the ROI above a limit. It is applied to locations known in position and size where a likely overheating is expected. This algorithm is preferentially used to protect movable probes or parts of the outboard limiters.

The second algorithm was implemented to detect hot spots in a larger ROI, e.g. local overheating of parts of the divertor. Here the expected hot spot is small compared to the divertor itself that is defined as a single ROI in each of the 3 video channels monitoring the divertor. This algorithm searches for the brightest $2 \times 2$ pixel square.

In addition to the selection of the active algorithm for each ROI, 4 levels of reaction by the CODAC system can be defined. At the moment the highest level is used to terminate the discharge by a soft pulse stop. But other actions are in preparation. e.g. for divertor protection: application of nitrogen seeding to increase the fraction of radiation in the divertor region and if this fails to protect the divertor, a reduction of the heating power. For probe protection: Moving the plasma away from the probe so that the distance of the reciprocating probe to the separatrix is increased.

In addition to the real-time monitoring the stored data are used for off-line analysis. (i) to reconstruct an event that was detected in real-time. (ii) to survey views and ROIs that are not included in the real-time safety system and (iii) to deliver input for physics investigation like arc detection and dust tracing.

\section{Implementation and experience from operation}

After a commissioning phase the VRT diagnostic was operated routinely during the past 1500 discharges. During this period 41 discharges were terminated by the VRT safety system. 15 discharges out of the 41 were false alarms because the system check in preparation of the discharge was not successful. For two shots the camera position was modified and a mismatch of the ROI results in a VRT safety discharge shutdown.

The remaining 26 discharges were terminated due to an overheating of components. In the following subsections two typical examples, one for each detection algorithm, are discussed.

Reflections at tungsten surfaces are not considered in the pre-processing and the detection algorithms. Nevertheless, no false alarm was caused by reflections. This is (i) because the monitored components are the hottest components in the vessel, so that reflection from colder components is negligible in particular in the vis/NIR wavelength region with the strong increase of the signal with temperature (see Fig. 2). And (ii) because 
the influence of specular reflection is excluded by proper settings for size and position of the ROI (Fig. 5)

\subsection{Integrated intensity}

The midplane manipulator is used to expose probes in the scrape-off layer plasma of ASDEX Upgrade. Fig. 5 shows an example for the exposition of a reciprocating probe that is moved $100 \mathrm{~mm}$ by a fast stroke. In the example the foremost position is $20 \mathrm{~mm}$ in front of the outboard limiter. The distance to the separatrix is about $30 \mathrm{~mm}$ and the heat load to the probe head is in the order of a few tens of $\mathrm{MW} / \mathrm{m}^{2}$. The temperature increase (hot spot intensity) is monitored by the VRT system. Due to the short exposure time of about $200 \mathrm{~ms}$, the safety limit is not reached and the stroke is performed once again. Due to the pre-heating of the probe, the resulting signal in the VRT safety system is higher. The limit is not exceeded and the discharge program runs as preprogrammed

\subsection{Hot spot in the divertor}

The whole outer divertor of AUG is protected by 3 video cameras locking from the top down into the divertor as shown in the inlet of Fig. 6 . The divertor tiles are tilted to avoid leading edges. Nevertheless damages of the tungsten coatings or dust can cause hot spots that have to be detected. Fig. 6 shows an example for a discharge with a hot spot. The intensity increases with heating power and time and results finally in a controlled ramp down of the discharge.

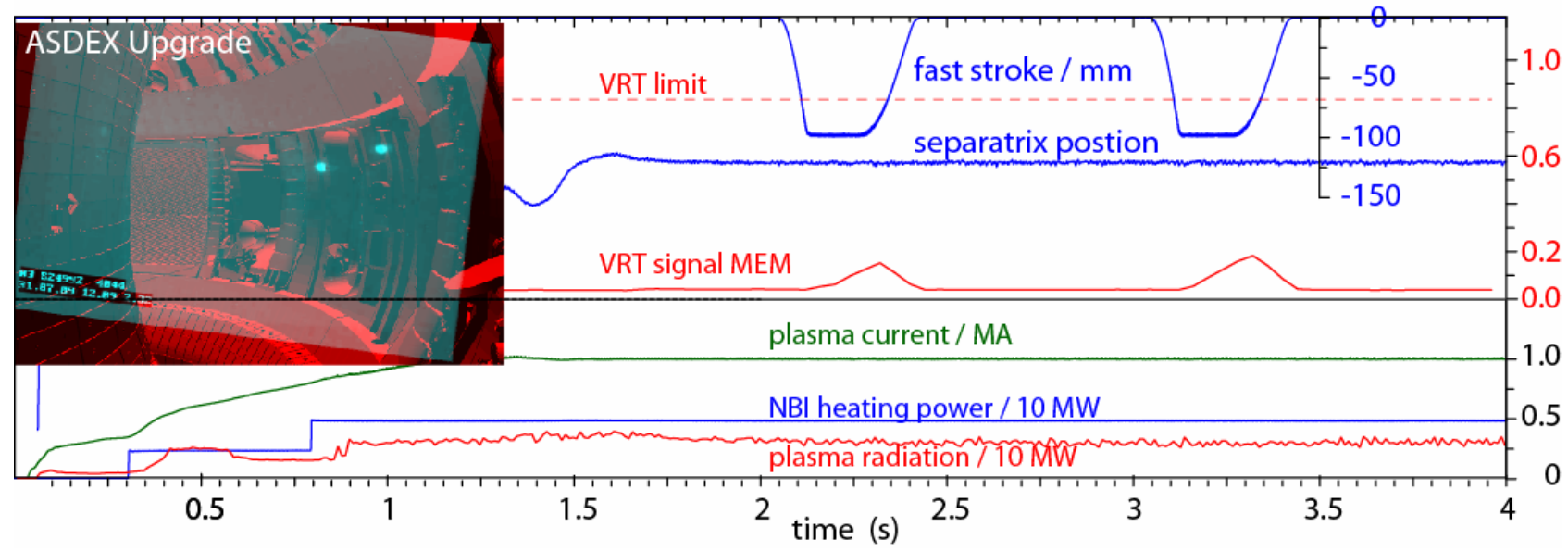

Fig. 5 AUG H-mode discharge with active safety algorithm for the midplane manipulator protection. The inlet shows a video image mapped to the in-vessel structure. The left hand side hot spot is the manipulator head. The right hand side spot is a reflection outside the ROI.

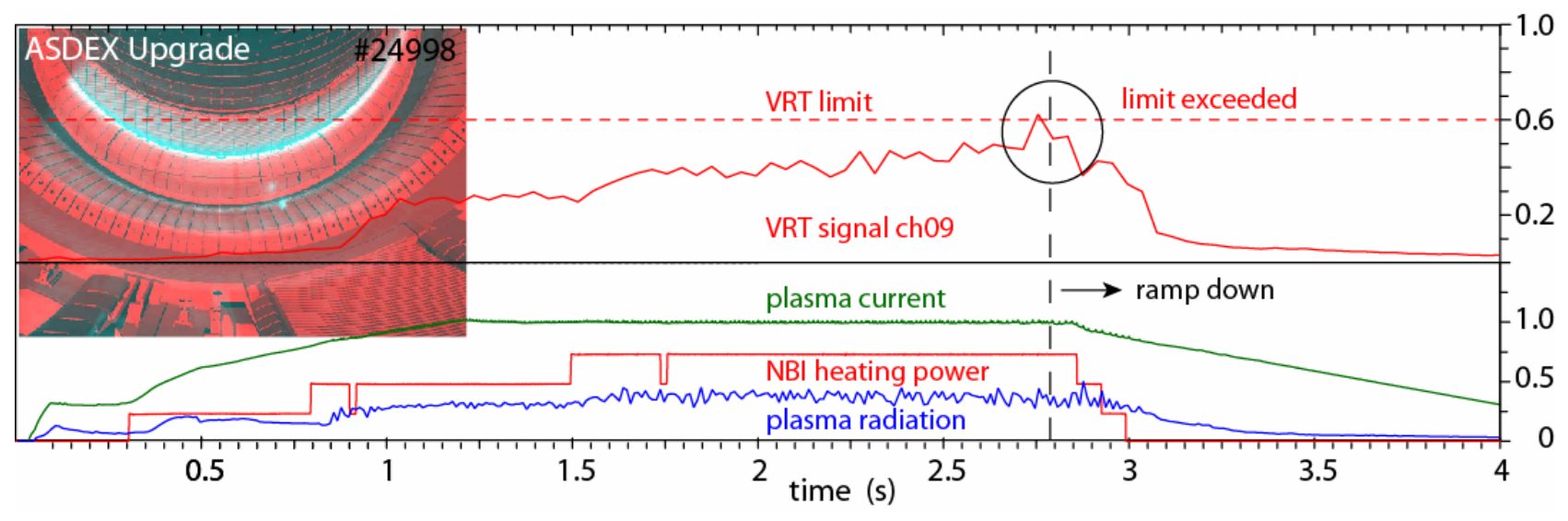

Fig. 6 AUG H-mode discharge with active safety algorithm for divertor protection. A hot spot in the divertor is detected @ $2.784 \mathrm{~s}$ and results in a ramp down of the discharge starting at $2.785 \mathrm{~s}$.

\section{Summary}

A video real time safety system (VRT) for protection of in-vessel components was fully implemented in the machine control system (CODAC) from the 2007 experimental campaign on. The VRT is based on video cameras instead of infrared systems. The visible wavelength range has a smaller measurement range but is a factor 5-10 more robust against changes of the optical system including emissivity and transmission compared to infrared systems. Up to 12 analog video channels with multiple regions of interest (ROI) are processed and monitored on each video stream. At present two safety algorithms, to detect the fraction of overheating in a ROI and hot spot detection, respectively, are implemented. The integral algorithm is preferentially used for probe or limiter protection, the hot spot algorithm for divertor protection. The VRT system is realized with ReadHawk real time operating system on a multi core 
Linux computer. The VRT system is operated routinely over 1500 discharges. $1 \%$ of these discharges were terminated by malfunction of the system. About $2 \%$ of the discharges were terminated due to the detection of hot spots.

\section{References}

[1] A. Herrmann (Guest editor), Fusion Science and Technology, 44 (2003) 1-747.

[2] A. Herrmann, et al., Phys. Scrip., T138 (2009).

[3] D. Guilhem, et al., Fusion Eng. Des., 74 (2005) 879-883.

[4] R. Reichle, et al., J. Nucl. Mater., 390-391 (2009) 10811085.

[5] G. Raupp, et al., Fusion Eng. Des., 81 (2006) 1747-1751.

[6] A. Herrmann, Optical surface temperature measurement, in: P.E. Stott, G. Gorini, E. Sindoni (Eds.) Diagnostics for Experimental Thermonuclear Fusion Reactors, Plenum Press, New York and London, 1996.

[7] R. Drube, to be published, (2010).

[8] http://www.ccur.com/redhawk realtime_linux_server.aspx 